\title{
Magnetic and Thermodynamic Properties of $\mathrm{Ce}_{4} \mathrm{RuAl}$
}

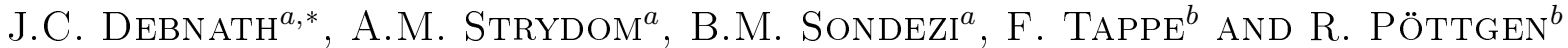 \\ ${ }^{a}$ Highly Correlated Matter Research Group, Physics Department, University of Johannesburg, \\ PO Box 524, Auckland Park 2006, South Africa \\ ${ }^{b}$ Institut für Anorganische und Analytische Chemie, Universität Münster, Germany \\ The results of magnetic susceptibility and heat capacity measurements are reported for the $\mathrm{Ce}_{4} \mathrm{RuAl}$ com- \\ pound above room temperature to low temperature range $(400 \mathrm{~K}$ to $0.34 \mathrm{~K}$ ) and in the magnetic field up to $7 \mathrm{~T}$. \\ The magnetic susceptibility $\chi(T)$ exhibits a distinct anomaly at $0.95 \mathrm{~K}$ which most probably suggests a paramag- \\ netic to antiferromagnetic phase transition. The magnetic susceptibility obeys the Curie-Weiss law in the region \\ $100-400 \mathrm{~K}$ and revealed an effective magnetic moment $\mu_{\mathrm{eff}}=2.18 \mu_{\mathrm{B}} / \mathrm{Ce}$ which is less than the value for free Ce $\mathrm{e}^{3+}$ \\ $\left(\mu_{\text {eff }}=2.54 \mu_{\mathrm{B}}\right)$. The paramagnetic Weiss temperature indicates net antiferromagnetic correlations. In the specific \\ heat a peak at $1.3 \mathrm{~K}$ supports the bulk nature of the phase transition observed in $\chi(T)$. The Sommerfield coeffi- \\ cient is moderately enhanced in the paramagnetic phase, and suggests $\mathrm{f}-\mathrm{c}$ correlations among the electrons prior to \\ magnetic ordering. The obtained Sommerfield coefficient $\gamma$ behavior is consistent with the Anderson model-based \\ theoretical predictions.
}

DOI: $10.12693 /$ APhysPolA.127.237

PACS: $75.30 . \mathrm{Cr}, 75.30 . \mathrm{Kz}, 75.30 . \mathrm{Mb}$

\section{Introduction}

Rare-earth elements are characterized by an incompletely filled $4 f$ shell, which is small compared to typical interatomic distances in metallic compounds and usually carry a stable and well defined magnetic moment even in metallic compounds. A large number of rareearth compounds exhibit unusual low-temperature properties, which are ascribed to interactions of the localized If moments with their environment. The crystal electric field $(\mathrm{CEF})$ at the rare-earth ion site splits the freeion ground-state multiplet. The temperature-dependent occupation probability of the CEF levels is reflected in many physical properties [1]. Intermetallic cerium compounds have widely been investigated in the last 40 years with respect to their intriguing physical properties [2]. This is due to the peculiar valence behavior, i.e. trivalent cerium has a $[\mathrm{Xe}]^{4} f^{1}$ configuration and exhibits paramagnetism (often accompanied by magnetic ordering), while tetravalent cerium, $[\mathrm{Xe}]^{4} f^{0}$, is diamagnetic. Various examples are known where the cerium valence can be influenced by temperature [3], by pressure [4], or upon hydrogenation [5].

As part of our program to explore new compounds to broaden our understanding of the physics of the strongly correlated electron class of materials, results of a variety of physical properties have been provided in this work to probe the magnetic and electronic behavior of the $\mathrm{Ce}_{4} \mathrm{RuAl}$ compound. To date, exploratory studies on $\mathrm{Ce}_{4} \mathrm{RuAl}$ have been reported at a conference [6] and by Tappe et al. [7]. In the present paper, we report the physical properties of the $\mathrm{Ce}-\mathrm{Ru}-\mathrm{Al}$ system, namely $\mathrm{Ce}_{4} \mathrm{RuAl}$. We have measured the susceptibility and magnetization of $\mathrm{Ce}_{4} \mathrm{RuAl}$ from $400 \mathrm{~K}$ to $0.5 \mathrm{~K}$.

\footnotetext{
* corresponding author; e-mail: jcd341@uowmail.edu.au
}

\section{Experimental}

The sample of $\mathrm{Ce}_{4} \mathrm{RuAl}$ was prepared by arc melting the stoichiometric amounts of the constituent elements on a water-cooled copper hearth in argon atmosphere. All the metals had a purity of $99.99 \%$. All the materials were melted at least four times and turned over after every melting to ensure homogeneous mixing. Powder X-ray diffraction was obtained using $\mathrm{Cu} K_{\alpha}$ radiation. Magnetization and heat capacity measurements were carried out using standard physical and magnetic measurement systems from Quantum Design (San Diego).

\section{Result and discussion}

Figure 1 shows the temperature dependence of magnetic susceptibility and inverse magnetic susceptibility $\chi^{-1}(T)$ between 1.76 to $400 \mathrm{~K}$ for $\mathrm{Ce}_{4} \mathrm{RuAl}$ measured in an applied field of $0.05 \mathrm{~T}$. A fit of the inverse magnetic susceptibility in the region $100-400 \mathrm{~K}$ according to a modified Curie-Weiss expression $\chi^{-1}(T)=\left[\chi_{0}+\right.$ $\left.C /\left(T-\theta_{P}\right)\right]^{-1}$ revealed a temperature-independent contribution $\chi_{0}=0.0003668 \mathrm{emu} /(\mathrm{Ce} \mathrm{mol})$, a magnetic moment $\mu_{\text {eff }}=2.18 \mu_{\mathrm{B}} / \mathrm{Ce}$, and the Weiss temperature $\theta_{P}=-81.33 \mathrm{~K}$.

This $\mu_{\text {eff }}$ value is higher than the obtained value by Tappe et al. [7]. According to Tappe et al. $\mu_{\text {eff }}$ value is $1.74 \mu_{\mathrm{B}} / \mathrm{Ce}$ and the $\theta_{P}=-34.1 \mathrm{~K}$ at applied field $B=$ $1 \mathrm{~T}$. At low temperatures, the inverse magnetic susceptibility $\chi^{-1}(T)$ considerably deviates from Curie-Weiss behaviour, which could be attributed to the crystallineelectric-field (CEF) effect. More experiments such as high-resolution angle-resolved photoemission studies and inelastic neutron scattering measurements are required to estimate the CEF parameters. The observed negative sign of $\theta_{P}$ can be understood to arise from the development of antiferromagnetic-type correlations between the Ce moments. 


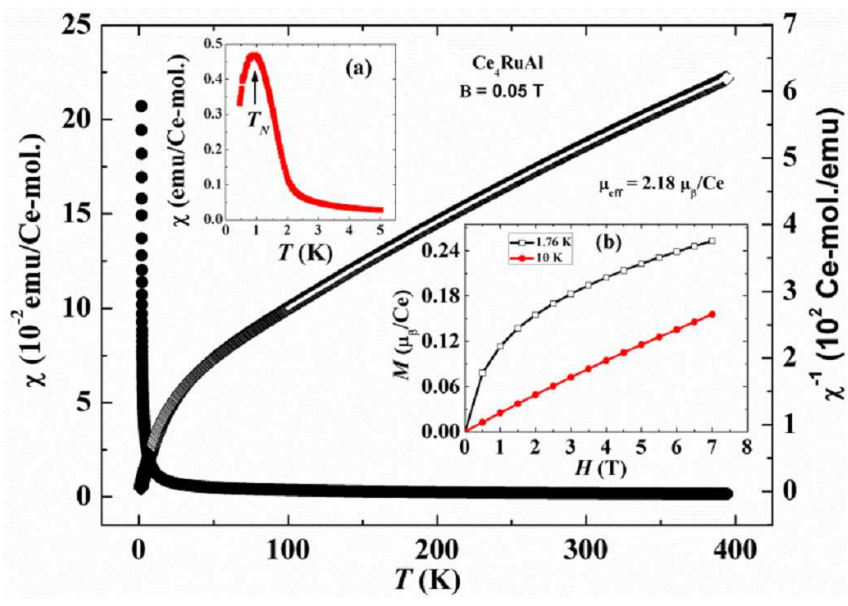

Fig. 1. Temperature dependence of the magnetic susceptibility and inverse susceptibility of $\mathrm{Ce}_{4} \mathrm{RuAl}$ measured in $0.05 \mathrm{~T}$. The line represents the modified CurieWeiss fit of $\chi^{-1}(T)$. Inset (a) shows the low temperature data of $\chi(T)$, arrow points to the anomaly corresponding to the magnetic phase transition. Inset (b) shows the magnetization for $\mathrm{Ce}_{4} \mathrm{RuAl}$ at different temperatures.

With decreasing temperature the magnetic susceptibility exhibits a distinct anomaly due to magnetic ordering at $T_{N}=0.95 \mathrm{~K}$. The inset (a) in Fig. 1 shows the low temperature dependence of $\chi(T)$ with better visible anomaly and the arrow indicating the magnetic phase transition.

The magnetic field dependence of magnetization at different temperatures for $\mathrm{Ce}_{4} \mathrm{RuAl}$ is presented in inset (b) of Fig. 1. The magnetization $M$ increases linearly with applied magnetic field at temperature $T=10 \mathrm{~K}$, whereas at $T=1.76 \mathrm{~K}$ on the other hand a strong curvature in $M(H)$ is found. It is noted that the magnetization is especially strongly curved and field dependent in the $\leq 2 \mathrm{~T}$ region. The value of magnetic moment measured at $1.76 \mathrm{~K}$ in a field of $7 \mathrm{~T}$ amounts to $0.25 \mu_{\mathrm{B}} / \mathrm{Ce}$ which is much lower than the theoretical value for the saturated moment of free $\mathrm{Ce}^{+3}$ ion $\mu_{\mathrm{s}}=2.14 \mu_{\mathrm{B}}$. It is probable that magnetocrystaline anisotropy plays an important role here. The obtained magnetic moment value is lower than the obtained value by Tappe et al. [7].

Figure 2 shows the temperature dependence of the heat capacity $C_{P}(T)$ of $\mathrm{Ce}_{4} \mathrm{RuAl}$ in the temperature range $0.5-300 \mathrm{~K}$ in zero magnetic field. The value of the electronic coefficient of the specific heat $\gamma$ has been taken as the extrapolation of the linear part of the $C_{P} / T$ vs. $T^{2}$ curves at low temperatures indicated by red line exposed in inset (c) of Fig. 2. The upturn in $C_{P} / T$ vs. $T^{2}$ observed at low temperatures is presumably related with the magnetic phase transition in low field[see inset (b) of Fig. 2]. The obtained electronic coefficient of specific heat $\gamma$ value is $158 \mathrm{~mJ} /\left(\mathrm{Ce} \mathrm{mol} \mathrm{K}{ }^{2}\right)$ for $\mathrm{Ce}_{4} \mathrm{RuAl}$.

This value of $\gamma$ suggests that there may be strong electronic correlations present in $\mathrm{Ce}_{4} \mathrm{RuAl}$. One origin may be the Kondo effect produced by an on-site hybridization between $f$ and conduction electrons. As a precursor effect to the lower-lying magnetic phase transition however,

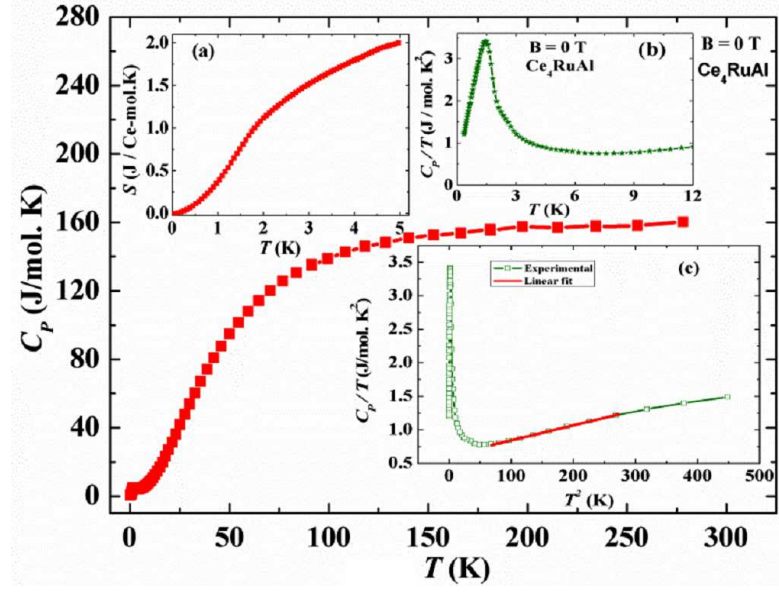

Fig. 2. Temperature dependence of the heat capacity of $\mathrm{Ce}_{4} \mathrm{RuAl}$ up to $300 \mathrm{~K}$ in zero magnetic field. Inset (a): The corresponding entropy as a function of temperature. Inset (b): Temperature dependence of the heat capacity displayed as $C_{P} / T$ Inset (c): a low temperature part of the heat capacity.

an enhanced value of $\gamma$ may also result from short-range correlations immediately above $T_{N}$. The value of $\theta_{D}$ obtained from the simplified Debye model is approximately $174 \mathrm{~K}$. Inset (c) of Fig. 2 shows the low-temperature part $(0.35 \mathrm{~K}$ to $12 \mathrm{~K})$ of the heat capacity $\left(C_{P} / T\right.$ vs. $\left.T\right)$ of $\mathrm{Ce}_{4} \mathrm{RuAl}$ in zero field.

We ascribe the sharp peak with a maximum at about $1.3 \mathrm{~K}$ in zero field, to the transition into a magnetically ordered phase. The appearance of the magnetic phase transition is consistent with measured susceptibility where strong maximum is present near this temperature shown in inset (a) in Fig. 1 where the magnetic susceptibility has a rapid decrease at $0.95 \mathrm{~K}$. The magnetic contribution to the specific heat and the corresponding magnetic entropy are related to the energy levels of the magnetic ions. In the absence of a nonmagnetic homologue with which to assess the lattice contribution, we show in inset (a) of Fig. 2 the total entropy of this system, which should be viewed as an upper limit of its $4 f$ magnetic entropy. At $T_{N}$ the obtained value of $S$ reaches about $14 \%$ for the case of a doublet ground state. The CEF effect of $\mathrm{Ce}^{3+}$ in cubic crystal symmetry is expected to split into one doublet and one quartet. Thus, our results support the view that even if all three site symmetries occupied by $\mathrm{Ce}$ in this structure, see [7], should participate in the magnetic ordering then the $\mathrm{Ce}^{3+}$ ions would still have to be in a strongly hybridized state to be concomitant to such a tiny fraction of the full available magnetic entropy of the $4 f$ spin system. Such a hybridized $\mathrm{Ce}^{3+}$ state in $\mathrm{Ce}_{4} \mathrm{RuAl}$ was in fact alluded to in the observed anomalous contraction of $\mathrm{Ce}_{4} \mathrm{RuAl}$ lattice parameter values in the $\mathrm{R}_{4} \mathrm{RuAl}$ series [7].

Figure 3 shows the low temperature part of $C_{P} / T$ vs. $T$ for $\mathrm{Ce}_{4} \mathrm{RuAl}$ in various applied magnetic fields. Due to the anomalous behavior at low temperatures the 


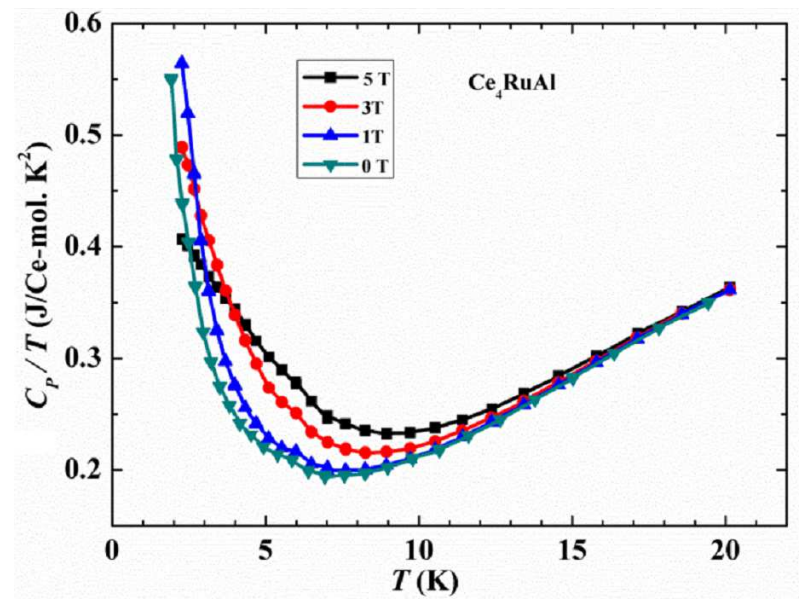

Fig. 3. Effect of magnetic field on the specific heat of $\mathrm{Ce}_{4} \mathrm{RuAl}$.

$\gamma$ coefficients value depend strongly on the temperature range used for the extrapolation and on the magnetic field. It evolves from the 158 to $180 \mathrm{~mJ} /\left(\mathrm{Ce} \mathrm{mol} \mathrm{K}{ }^{2}\right)$ for $(\mathrm{H})$ increasing from 0 to $5 \mathrm{~T}$. The obtained $\gamma$ behavior is consistent with the Anderson model-based theoretical predictions [8].

\section{Conclusions}

Magnetic susceptibility with paramagnetic to antiferromagnetic phase transition and heat capacity measurements suggest that there may be strong electronic correlations present in $\mathrm{Ce}_{4} \mathrm{RuAl}$. The heat capacity measurements provide $\gamma=158 \mathrm{~mJ} /\left(\mathrm{Ce} \mathrm{mol} \mathrm{K}^{2}\right.$ for the electron specific heat coefficient showing that there is an enhancement of the effective mass. The enhanced value of $\gamma$ originates from an appreciable amount of $f$-electron involvement in the conduction electrons and the resulting strongly hybridized state. The obtained $\gamma$ behavior for different applied fields is consistent with the Anderson model-based theoretical predictions.

\section{Acknowledgments}

J.C.D. acknowledges the NRF (Grant no. 85115) for an innovation Postdoctoral Research Fellowship, supervised by A.M.S. A.M.S. thanks the SA-NRF (78832) and the URC of UJ for financial assistance.

\section{References}

[1] P. Coleman, in: Handbook of Magnetism and Advanced Magnetic Materials, Vol. 1, Eds. H. Knoemuller, S. Parkin, Wiley, New York 2007.

[2] A. Szytuła, J. Leciejewicz, Handbook of Crystal Structures and Magnetic Properties of Rare Earth Intermetallics, 2nd ed., CRC Press, Boca Raton 1994.

[3] T. Ueda, D. Honda, T. Shiromoto, N. Metoki, F. Honda, K. Kaneko, Y. Haga, T.D. Matsuda, T. Takeuchi, A. Thamizhavel, K. Sugiyama, K. Kindo, R. Settai, Y. Onuki, J. Phys. Soc. Jpn. 74, 2836 (2005).

[4] A. Jayaraman, W. Lowe, L.D. Longinotti, E. Bucher, Phys. Rev. Lett. 36, 366 (1976).

[5] B. Chevalier, R. Decourt, B. Heying, F.M. Schappacher, U.Ch. Rodewald, R.-D. Hoffmann, R. Pöttgen, R. Eger, A. Simon, Chem. Mater. 19, 28 (2007).

[6] Z.M. Kurenbaeva, E.V. Murashova, D.N. Hannanov, A.B. Ilyukin, A.I. Tursina, Y.D. Seropegin, in: XIth Int. Conf. on Crystal Chemistry of Intermetallic Compounds, Lviv (Ukraine), Ivan Franko National University of Lviv, Lviv 2010, p. 61.

[7] F. Tappe, C. Schwickert, S. Linsinger, R. Pöttgen, Monatsh Chem. 142, 1087 (2011).

[8] J.W. Kim, J. Callaway, D.P. Chen, Phys. Rev. B 47, 2890 (1993). 